Disponível em

http://www.anpad.org.br/rac

RAC, Rio de Janeiro, v. 21, n. 1, art. 3, pp. 41-61, Jan./Fev. 2017

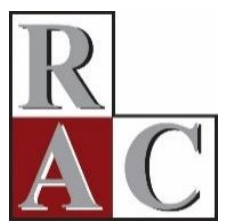

$(\mathrm{ccc}) \mathrm{EY}$

\title{
Atuação do Líder na Gestão Estratégica de Pessoas: Reflexões, Lacunas e Oportunidades
}

A Leader's Role in Strategic People Management: Reflections, Gaps and Opportunities

Eliane Maria Pires Giavina Bianchi ${ }^{1}$ Alessandra Quishida ${ }^{2}$ Paula Gabriela Foroni ${ }^{2}$

Faculdade Campo Limpo Paulista ${ }^{1}$ Universidade de São Paulo ${ }^{2}$

Artigo recebido em 09.10.2015. Última versão recebida em 13.06.2016. Aprovado em 14.06.2016. Publicado online em 05.09.2016. 


\title{
Resumo
}

A gestão estratégica de pessoas é um tema bastante estudado, enfatizando a relação entre políticas e práticas de gestão e outras variáveis organizacionais. A atuação dos atores organizacionais na gestão é pouco trabalhada, tornando o processo de implementação da gestão de pessoas pouco explorado. $\mathrm{O}$ estudo sobre a área de recursos humanos contribui para evidenciar uma lacuna na implementação, pois enfatiza o papel consultivo dos profissionais. Este trabalho objetiva analisar a atuação dos líderes como elo na gestão estratégica de pessoas por meio de reflexões sugeridas e pautadas em referencial teórico (gestão estratégica de pessoas, liderança e relações entre essas temáticas) e sugerir uma integração de modelos (liderança e gestão de pessoas) para suportar a evolução dos estudos. As quatro orientações do líder propostas por Yukl (2012), as definições de espaço organizacional de O'Reilly, Caldwell, Chatman, Lapiz e Self (2010), bem como o modelo tridimensional de Gratton e Truss (2003) e a proposta de papéis na gestão de pessoas de Ulrich (1997) podem ser base para a integração dos temas. Por meio deste artigo, pretende-se contribuir fornecendo sustentação para o desenvolvimento e o aprofundamento de estudos empíricos, além de endereçar uma lacuna sobre a temática da implementação na gestão estratégica de pessoas.

Palavras-chave: gestão estratégica de pessoas; liderança; papéis organizacionais; alinhamento; implementação estratégica.

\begin{abstract}
Strategic people management is a widely studied topic, emphasizing the relationship between management policies and practices and other organizational variables. The managerial roles of organizational actors have been scarcely researched, making the implementation process of people management little explored. The study of human resources contributes to show a gap in the implementation process, as it addresses the role of professional consultants. This work aims to analyze the performance of leaders as a link in strategic people management through reflections suggested and guided by theoretical framework (strategic people management, leadership and the relations between those themes) and suggests an integration of theoretical models (leadership and people management) to support the evolution of research. The four leader orientations proposed by Yukl (2012), the organizational spaces defined by O'Reilly, Caldwell, Chatman, Lapiz and Self (2010), as well as Gratton and Truss' (2003) three-dimensional model and the people management roles Ulrich (1997) suggests may serve as a base to integrate the themes. Through this article, we intend to contribute by providing support for the development and deepening of empirical studies, as well as address the topic of implementation in strategic management of people.
\end{abstract}

Key words: strategic people management; leadership; organizational roles; organizational fit; strategic implementation. 


\section{Introdução}

A gestão estratégica de pessoas é uma temática bastante presente nos estudos em administração. Desde a década de 1980, com a aparição dos primeiros estudos na área (Miles \& Snow, 1984; Tichy, Fombrun, \& Devanna, 1982), os pesquisadores enveredaram pelas mais diversas arenas: busca de fundamentação teórica para a gestão de pessoas (Watson, 2007; Wright \& McMahan, 1992); desenvolvimento de perspectivas teóricas para a pesquisa em gestão de pessoas (Delery \& Doty, 1996; Martín-Alcázar, Romero-Fernández, \& Sánchez-Gardey, 2005); entendimento da evolução da temática por meio da análise histórica (Fischer, 2002; Jamrog \& Overholt, 2004); posicionamento da área e dos profissionais de recursos humanos (Baron \& Kreps, 1999; Lawler, 2005; Ulrich, 1997, 2004); ou a relação da gestão estratégica de pessoas com resultados organizacionais (Datta, Guthrie, \& Wright, 2005; Pfeffer, 1994; Wright, Gardner, Moynihan, \& Allen, 2005); entre outras. Observa-se, pois, que a Gestão de Pessoas é discutida no campo teórico e no empírico.

Diferentemente de estudiosos de estratégia organizacional, que evoluíram suas análises da formulação estratégica para a implementação estratégica (Hrebiniak, 2006; Thompson \& Strickland, 1996), os trabalhos em gestão de pessoas parecem estar mais focados em filosofias, políticas, programas, práticas e processos - formulação (Martín-Alcázar et al., 2005; Saá-Pérez \& Garcia-Falcón, 2011; Veloso et al., 2014) do que na implementação da gestão de pessoas - papéis e relações organizacionais existentes nessa gestão. Quando enfatizam os papéis organizacionais, o olhar é centrado nos profissionais de recursos humanos, pontuando que estes devem ter atuação estratégica e consultiva. Essa situação sugere uma lacuna no processo de execução/implementação da gestão de pessoas. Muito recentemente, alguns estudiosos começaram a refletir sobre o gerente de linha como um mediador da percepção das práticas de gestão (Alfes, Truss, Soane, Rees, \& Gatenby, 2013; Bos-Nehles, Riemsdesjk, \& Looise, 2013; Flickinger, Allscher, \& Fiedler, 2016; Kelliher, Hailey, \& Farndalde, 2013; Lakshman, 2014), mas os estudos ainda são incipientes e, algumas vezes, enfatizam uma única prática de gestão.

Este trabalho ${ }^{(1)}$ objetiva analisar a atuação do líder como elo fundamental na gestão estratégica de pessoas, por meio de reflexões sugeridas e pautadas em referencial teórico. Trata-se, portanto, de um ensaio teórico que analisa as temáticas de gestão estratégica de pessoas, liderança, e a relação entre esses dois temas para entender a estágio atual dos conceitos e estudos, e levantar oportunidades, lacunas e proposições para estudos futuros. O uso do termo liderança, neste trabalho, pretende ir além do perfil, reforçando o exercício do papel do líder. Espera-se contribuir para a evolução no campo da gestão estratégica de pessoas, à luz dos autores de estratégia organizacional.

\section{Gestão Estratégica de Pessoas: Fundamentação, Perspectivas Teóricas e Campo de Estudo}

A partir da década de 1980, a preocupação com a orientação dos comportamentos dos indivíduos para o atingimento dos objetivos organizacionais foi o que evidenciou o caráter estratégico da gestão de pessoas para os negócios. O termo Gestão Estratégica de Pessoas está relacionado às visões comportamental e normativa (Legge, 2005). Enfatizando a visão normativa, Martín-Alcázar et al. (2005) definem gestão estratégica de pessoas como "um conjunto integrado de práticas, políticas e estratégias por meio das quais as organizações gerenciam seu capital humano, que influenciam e são influenciadas pela estratégia do negócio, pelo contexto organizacional e pelo contexto socioeconômico" (p. 651). Sob a perspectiva comportamental, para Armstrong (2011), a gestão estratégica de pessoas é "uma abordagem para gerenciar pessoas que lida com as maneiras pelas quais os objetivos organizacionais são alcançados por seus recursos humanos, por intermédio de estratégias, políticas e práticas integradas de RH” (p. 48). Boxall e Purcell (2003), e Armstrong (2011) ainda enfatizam o senso de direção e a satisfação de necessidades organizacionais, individuais e coletivas dos profissionais, íncluindo atores organizacionais no processo de gestão. Não se pode esquecer, também, evidenciando o caráter 
estratégico, o ambiente de negócio em que a organização está inserida, seu contexto competitivo e suas capacidades (Albuquerque, 2002; Gratton \& Truss, 2003; Ulrich, 2004).

\section{Perspectivas teóricas em gestão de pessoas}

O estudo da gestão de pessoas se apoia em conceitos teóricos da psicologia, da economia, de finanças, da estratégia, entre outras, como sugere Watson (2007), e não desenvolveu sua própria teoria. Em muitos casos, os trabalhos são estudos empíricos, sem fundamentação teórica (Wright \& McMahan, 1992). Por isso, um esforço de articulação teórica foi realizado por alguns autores. Wright e McMachan (1992), Lepak e Snell (1999) e Watson (2007), de formas diferentes, buscaram linhas teóricas ou mesmo teorias para suportar modelos de gestão ou implicações para a gestão de pessoas. A Tabela 1 apresenta uma reflexão sobre as implicações - organizacionais e na gestão de pessoas - resultantes de possíveis articulações teóricas.

Tabela 1

\section{Reflexões sobre a Teorização na Gestão de Pessoas}

\begin{tabular}{|c|c|c|}
\hline BaseTeórica & Visão Organizacional & Implicação para a Gestão de Pessoas \\
\hline $\begin{array}{l}\text { Linha } \\
\text { Funcionalista/Sistemas } \\
\text { Cibernéticos }\end{array}$ & $\begin{array}{l}\text { Organização é vista como um sistema } \\
\text { fechado (recebe as influências do } \\
\text { ambiente) ou aberto (interage com o } \\
\text { ambiente). }\end{array}$ & $\begin{array}{l}\text { Se a organização é um sistema aberto, a } \\
\text { empresa recebe demandas do ambiente } \\
\text { (necessidades e oportunidades), processa } \\
\text { essas demandas em conformidade com } \\
\text { suas políticas e práticas (gerenciando } \\
\text { comportamentos e competências) e, como } \\
\text { saída, produz desempenho organizacional } \\
\text { e desenvolvimento humano. O dinamismo } \\
\text { do processo representa uma oportunidade } \\
\text { para os indivíduos. }\end{array}$ \\
\hline Linha Werberiana & $\begin{array}{l}\text { Organização é vista como espaço de } \\
\text { rivalidades, conflitos de interesse e poder. }\end{array}$ & $\begin{array}{l}\text { A organização não consegue ter clareza } \\
\text { das políticas e práticas de Gestão de } \\
\text { Pessoas, pois as relações de poder } \\
\text { continuamente afetam a implementação } \\
\text { dessas práticas, por meio dos conflitos e } \\
\text { das relações de influência. O foco da } \\
\text { gestão pode ser, ainda, administrar } \\
\text { interesses individuais que muitas vezes se } \\
\text { disfarçam em objetivos organizacionais. }\end{array}$ \\
\hline $\begin{array}{l}\text { Linha Marxiniana/ } \\
\text { Teoria do Custo da } \\
\text { Transação }\end{array}$ & $\begin{array}{l}\text { Organização é vista como uma máquina } \\
\text { com processos, controles e monitoramento } \\
\text { de resultados bem definidos. Também } \\
\text { existe a definição clara de troca entre as } \\
\text { diversas partes. }\end{array}$ & $\begin{array}{l}\text { A gestão de pessoas implica na } \\
\text { manutenção da máquina em } \\
\text { funcionamento, realizando os processos } \\
\text { tradicionais de gestão de pessoas } \\
\text { (recrutamento, treinamento, } \\
\text { remuneração...). Pode, ainda, representar } \\
\text { uma relação despersonificada, baseada } \\
\text { fundamentalmente em direitos e deveres. }\end{array}$ \\
\hline $\begin{array}{l}\text { Linha Pós- } \\
\text { Estruturalista/ Teoria } \\
\text { Institucional }\end{array}$ & $\begin{array}{l}\text { Organização é vista como uma entidade } \\
\text { regida por discursos que representam uma } \\
\text { verdade aprendida em determinado } \\
\text { momento. }\end{array}$ & $\begin{array}{l}\text { A gestão de pessoas seria afetada pela } \\
\text { história, pela cultura, pelas verdades } \\
\text { incorporadas, podendo ser, até certo } \\
\text { ponto, bastante estática. }\end{array}$ \\
\hline
\end{tabular}


Tabela 1 (continuação)

\begin{tabular}{lll}
\hline BaseTeórica & Visão Organizacional & Implicação para a Gestão de Pessoas \\
\hline $\begin{array}{l}\text { Teoria dos Recursos } \\
\text { da Firma }\end{array}$ & $\begin{array}{l}\text { A organização deve se diferenciar a partir } \\
\text { de seus recursos de valor, únicos e difíceis } \\
\text { de serem imitados. }\end{array}$ & $\begin{array}{l}\text { As pessoas podem ser recursos } \\
\text { viabilizadores ou limitadores da } \\
\text { implementação da estratégia } \\
\text { organizacional. Se viabilizadores, elas são } \\
\text { fonte de vantagem competitiva. }\end{array}$ \\
$\begin{array}{l}\text { Perspectiva } \\
\text { Comportamental }\end{array}$ & $\begin{array}{l}\text { A organização deve se preocupar em } \\
\text { alinhar a estratégia organizacional e as } \\
\text { estratégias funcionais evidenciando uma } \\
\text { busca por comportamentos direcionados. }\end{array}$ & $\begin{array}{l}\text { A busca de alinhamento, como dinâmica } \\
\text { organizacional, favorece a elaboração de } \\
\text { desafios e desenvolvimento para as } \\
\text { pessoas. }\end{array}$ \\
\hline
\end{tabular}

Nota. Fonte: elaborada com base em Wright, P. M., \& McMahan, G. C. (1992). Theoretical perspectives for strategic human resource management. Journal of Management, 18(2), 295-320. http://dx.doi.org/10.1177/014920639201800205; Lepak, D. P., $\&$ Snell, S. A. (1999). The human resource architecture: toward a theory of human capital allocation and development. Academy of Management Review, 24(1), 31-48. http://dx.doi.org/10.5465/AMR.1999.1580439; Watson, T. (2007). Organization theory and HRM. In P. Boxall, J. Purcell, \& P. Wright (Orgs.), The Oxford handbook of human resource management (pp. 108-127). New York: Oxford University Press.

Apesar de todas as bases teóricas possibilitarem reflexões e demonstrarem implicações na forma de perceber a organização e possíveis consequências para a gestão de pessoas, percebe-se que nem todas as fundamentações sustentam o conceito de Gestão Estratégica de Pessoas. A Teoria Funcionalista, a Teoria dos Recursos da Firma e a Perspectiva Comportamental são as fundamentações que mais suportam questões como direcionamento de comportamento, atingimento de resultados e organização de processos de forma articulada, além de poscionar o indivíduo como um recurso/ator organizacional importante.

Outro insumo trazido pela busca de fundamentação teórica é que a gestão de pessoas não implica somente relação entre estas, mas, principalmente, relações entre processos e entre pessoas e processos.

Ainda questionando o embasamento teórico na gestão de pessoas, mas optando por uma visão mais aplicada, Delery e Doty (1996) trabalharam no que denominaram de perspectivas teóricas para gestão de pessoas, classificando-as em: universalista, contingencial e configuracional. Essas perpectivas fundamentalmente suportam a definição de estudos empíricos e não se relacionam com as bases teóricas mencionadas anteriormente. Martín-Alcázar, Romero-Fernández e Sánchez-Gardey (2005) adicionaram a estas a perpectiva contextual. A Tabela 2 apresenta uma discussão sobre as perpectivas. 
Tabela 2

\section{Comparativo entre as Perspectivas Teóricas que Fundamentam o Estudo da Gestão de Pessoas}

\begin{tabular}{|c|c|c|c|}
\hline Perspectiva & Premissas Básicas & $\begin{array}{l}\text { Relação entre as práticas de } \\
\text { gestão de pessoas, a } \\
\text { estratégia do negócio e o } \\
\text { ambiente }\end{array}$ & $\begin{array}{l}\text { Considerações para } \\
\text { utilização }\end{array}$ \\
\hline Universalista & $\begin{array}{l}\text { Pressupõe a utilização de } \\
\text { melhores práticas de gestão } \\
\text { de pessoas. }\end{array}$ & $\begin{array}{l}\text { Não existe. As práticas são } \\
\text { consideradas } \\
\text { autossuficientes. }\end{array}$ & $\begin{array}{l}\text { Cada prática, isoladamente, } \\
\text { leva a uma performance } \\
\text { organizacional superior, } \\
\text { independentemente do } \\
\text { contexto, segmento de } \\
\text { atuação da organização ou } \\
\text { outras práticas de gestão. }\end{array}$ \\
\hline Contingencial & $\begin{array}{l}\text { Não existem melhores } \\
\text { práticas. O efeito das práticas } \\
\text { de gestão de pessoas é } \\
\text { dependente da estratégia } \\
\text { organizacional ou do } \\
\text { ambiente (interno e externo). }\end{array}$ & $\begin{array}{l}\text { As práticas são definidas em } \\
\text { função das opções } \\
\text { organizacionais, em especial } \\
\text { a estratégia. }\end{array}$ & $\begin{array}{l}\text { Muitas contingências internas } \\
\text { e externas podem afetar a } \\
\text { definição ou o resultado de } \\
\text { uma ou de um conjunto de } \\
\text { práticas. A interação entre as } \\
\text { práticas é pouco considerada. }\end{array}$ \\
\hline Configuracional & $\begin{array}{l}\text { É possível estabelecer um } \\
\text { conjunto de práticas, definido } \\
\text { como um sistema de RH, que } \\
\text { se inter-relaciona de } \\
\text { múltiplas formas. }\end{array}$ & $\begin{array}{l}\text { A integração entre as práticas } \\
\text { do conjunto pode ser mais } \\
\text { importante do que as relações } \\
\text { com as opções } \\
\text { organizacionais. }\end{array}$ & $\begin{array}{l}\text { Determinados conjuntos de } \\
\text { práticas (sistemas de } \mathrm{RH} \text { ) } \\
\text { podem atender e/ou } \\
\text { responder às demandas } \\
\text { específicas do ambiente de } \\
\text { negócio. }\end{array}$ \\
\hline Contextual & $\begin{array}{l}\text { O sistema de RH é } \\
\text { considerado parte de um } \\
\text { macro ambiente, recebendo e } \\
\text { fornecendo influência deste. }\end{array}$ & $\begin{array}{l}\text { A definição do sistema de } \\
\text { RH considera contextos de } \\
\text { negócio e posicionamento } \\
\text { organizacional. }\end{array}$ & $\begin{array}{l}\text { O desempenho } \\
\text { organizacional é uma } \\
\text { condição multidirecional, } \\
\text { dependente de relações } \\
\text { sinérgicas entre múltiplas } \\
\text { variáveis. }\end{array}$ \\
\hline
\end{tabular}

Nota. Fonte: Adaptada de Martín-Alcázar, F., Romero-Fernández, P. M., \& Sánchez-Gardey, G. (2005). Strategic human resource management: integrating the universalistic, contigent, configurational and contextual perspectives. The International Journal of Human Resource Management, 16(5), 633-659. http://dx.doi.org/10.1080/09585190500082519

Observa-se, na Tabela 2, que todas as perspectivas tratam de relações entre processos (estratégias, contingências ou práticas) acrescentando mais ou menos variáveis, dependendo da complexidade e foco do estudo do pesquisador. Silveira (2014) ainda compara o alinhamento estratégico de gestão de pessoas (relações entre variáveis) com a discussão de alinhamento trazida pelos estudiosos da estratégia que, apesar de usar nomenclaturas diferentes, utilizam os mesmos processos discutidos anteriormente relação entre estratégias, contingências e práticas.

\section{Campo de estudo em gestão de pessoas}

Apesar da preocupação com a fundamentação teórica, poucos são os trabalhos empíricos que a mencionam. Por outro lado, as perspectivas teóricas são sempre base dos estudos. Uma boa parte dos trabalhos utiliza a perspectiva universalista e tem como principal preocupação os resultados organizacionais, sejam estes financeiros ou outros indicadores de negócio. Os trabalhos buscam, analisando resultados consistentes, listar as melhores práticas que suportaram esses resultados (ex: Collins \& Porras, 1997; Delaney \& Huselid, 1996; Pfeffer, 1994, 1998) ou relacionar práticas a indicadores, mostrando sua importância (ex: Batt, 2002; Datta et al., 2005; Ventura \& Leite, 2014). Outros, ainda, propõem modelos para a análise de melhores práticas organizacionais (Bassi \& McMurrer, 2007; Demo, Neiva, Nunes, \& Rozett, 2012). 
Sob a perspectiva contingencial, pesquisadores procuram verificar se determinadas estratégias se relacionam com práticas de gestão. Alguns modelos (Albuquerque, 2002; Arthur, 1992; Schuler \& Jackson, 1987) suportam uma série de trabalhos que busca verificar o alinhamento entre estratégia organizacional e práticas de gestão de pessoas (ex: Bianchi, 2008; Foroni, 2014; Lee, 1994). Esses trabalhos, na maioria das vezes, percebem que o alinhamento é uma abordagem dinâmica, como sugerem Wright e Snell (1998). O uso da perspectiva contingencial aborda, mesmo que de forma não explícita, a questão do comportamento humano direcionado e gerenciado.

Pode-se até dizer que os modelos mencionados para suportar os trabalhos na linha contingencial também apresentem configurações (sistemas de práticas), mas na maioria das vezes em que são utilizados relacionam as práticas à estratégia. Dentre os pesquisadores que relacionaram conjunto de práticas a resultados, pode-se citar Huselid (1995). Lepak e Snell (1999, 2002) mostram que o conjunto de práticas pode também ter relações com o mercado de trabalho e com formas de vínculos organizacionais. Ostroff e Bowen (2016) ainda argumentam que a eficácia de um conjunto de práticas está relacionada à sua visibilidade, à consistência interna, e à consistência entre intenção e execução. Os trabalhos da linha configuracional sugerem que, muitas vezes, a temática é mais complexa, e uma organização vai precisar arquitetar vários sistemas de práticas para seus diversos públicos internos ou mesmo considerar um maior número de variáveis organizacionais para sua definição (ex: Jiang et al., 2012; Liu, Gong, Zhou, \& Huang, in press).

Sob a perspectiva contextual, encontram-se poucos trabalhos. Porém, alguns estudos que analisam configurações e se preocupam com contingências organizacionais internas e externas estariam sendo realizados sob essa perspectiva. As perspectivas revelam o ângulo de análise do pesquisador, a maneira pela qual ele faz a leitura do fenômeno em estudo. Cabe salientar que, quanto mais integrações de variáveis se fizerem, mais estratégicas podem ser as análises. Todas as perspectivas buscam salientar o sucesso organizacional e algumas práticas de gestão, como desenvolvimento, reconhecimento e responsabilização das pessoas aparecem quase sempre nos resultados dos trabalhos aqui discutidos, não importando a perspectiva de estudo utilizada.

Wright e Snell (1998) ainda discutem os termos alinhamento e flexibilidade para representar a atuação organizacional quando considerada a gestão de pessoas. A organização precisa garantir coerência de atuação perante as pessoas (alinhamento) e também tempo adequado de respostas para demandas do contexto (flexibilidade). Ainda, a aplicação de uma prática de gestão pode ser isolada, mas o resultado das mesmas são atingidos em conjunto, uma vez que os funcionários são expostos a todas elas simultaneamente (Wei, 2006; Wright \& Boswell, 2002).

Outra questão a ser debatida é a relação entre a intenção e a percepção das práticas de gestão de pessoas. Muitas vezes, mesmo que de forma não explícita, essa questão aparece nos resultados de pesquisa. As diferenças entre intenção e percepção permeiam a execução/implementação na gestão de pessoas, tema estudado ainda de forma fragmentada.

\section{Líderes e Gestão Estratégica de Pessoas: Relações Atuais e Relações Possíveis}

Além dos profissionais de recursos humanos, dois outros atores são fundamentais na implementação da gestão de pessoas: os funcionários, pacientes do processo, e os gestores, muitas vezes executores principais dos processos. Nesse sentido, a liderança (relação entre líder e liderado) ou mesmo o exercício do papel organizacional do líder/gestor, torna-se um assunto crítico a ser discutido.

Esse estudo opta por discutir o líder e não o gestor como ator organizacional importante na execução da gestão de pessoas. A origem do conceito de gerente é anterior ao conceito de líder. O papel do gerente, inicialmente definido com base nas funções da administração - planejar, organizar, dirigir e controlar (Maximiano, 2004; Pereira, Albuquerque Maranhão, Rezende, \& Mendonça, 2015), evoluiu e recebeu atribuições relacionadas às pessoas com as quais este (o gerente) se relacionava. Por outro lado, 
o conceito de líder, que tratava de características individuais e das relações pessoais, recebeu uma perspectiva mais completa, passando a relacionar outras variáveis contingenciais (Day \& Harrison, 2007; Yukl, 2012). Dessa forma, hoje, os conceitos podem até se confundir quando observados no campo organizacional (Maximiano, 2004; Motta \& Vasconcelos, 2004; Spisak, O’Brien, Nicholson, \& Van Vugt, 2015), mas a investigação sobre o líder é mais contemporânea, mais ampla do que o estudo sobre gestores e mais suportada por ciências diversas, em especial quando relacionado à temática de recursos humanos.

\section{Liderança: conceitos e modelos}

Liderança é o principal arcabouço teórico para a discussão sobre o líder. Embora o fenômeno da liderança seja tradicionalmente pesquisado e publicado, sua complexidade e multidimensionalidade dificultam a possibilidade de um consenso, visão também corroborada por Day e Harrison (2007). A Tabela 3 apresenta alguns conceitos desenvolvidos desde a década de 1950.

Tabela 3

\section{Conceitos de Liderança}

\begin{tabular}{|c|c|}
\hline Conceitos & Fontes \\
\hline $\begin{array}{l}\text { "Liderança é o comportamento de um indivíduo quando está dirigindo as } \\
\text { atividades de um grupo que segue rumo a um objetivo comum." }\end{array}$ & $\begin{array}{l}\text { Hemphill e Coons (1957, p. } 7 \\
\text { como citado Bergamini, 2009, } \\
\text { p. 3). }\end{array}$ \\
\hline $\begin{array}{l}\text { "Liderança é o tipo especial de relacionamento de poder, caracterizado pela } \\
\text { percepção dos membros do grupo, no sentido de que outro membro tenha o } \\
\text { direito de prescrever padrões de comportamento no que diz respeito à } \\
\text { qualidade de membro desse grupo." }\end{array}$ & $\begin{array}{l}\text { Janda }(1960, \text { p. } 358 \text { como } \\
\text { citado Bergamini, 2009, p. 3). }\end{array}$ \\
\hline $\begin{array}{l}\text { "Liderança é uma interação entre pessoas, na qual uma apresenta informação } \\
\text { de um tipo e de tal maneira que os outros se tornam convencidos que seus } \\
\text { resultados ... serão melhorados caso se comporte da maneira sugerida." }\end{array}$ & $\begin{array}{l}\text { Jacobs }(1970, \text { p. } 232 \text { como } \\
\text { citado Bergamini, 2009, p. 3). }\end{array}$ \\
\hline $\begin{array}{l}\text { "Liderança é o processo de influenciar as atividades de um grupo organizado } \\
\text { na direção da realização de um objetivo." }\end{array}$ & $\begin{array}{l}\text { Roach e Behling (1984, p. } 46 \\
\text { como citado Bergamini, } 2009 \text {, } \\
\text { p. 3). }\end{array}$ \\
\hline $\begin{array}{l}\text { "Liderança é um processo de influência que também reconhece que os líderes } \\
\text { podem influenciar os membros do grupo por meio de seu próprio exemplo." }\end{array}$ & $\begin{array}{l}\text { Stogdill (1990, p. } 15 \text { como } \\
\text { citado Bergamini, 2009, p. 3). }\end{array}$ \\
\hline $\begin{array}{l}\text { "Liderança é uma relação entre aqueles que aspiram a ela e aqueles que } \\
\text { escolheram segui-la." }\end{array}$ & Kouzes e Posner (1997, p. 98). \\
\hline $\begin{array}{l}\text { Liderança é a "capacidade de influenciar um conjunto de pessoas para alcançar } \\
\text { metas e objetivos." }\end{array}$ & $\begin{array}{l}\text { Robbins, Judge e Sobral } \\
(2010, \text { p. 359). }\end{array}$ \\
\hline
\end{tabular}

Nota. Fonte: Elaborada com base em Bergamini, C. W. (2009). Liderança: administração do sentido. São Paulo: Atlas. Kouzes, J. M., \& Posner, B. Z. (1997). O desafio da liderança. Rio de Janeiro: Campus. Robbins, S. P., Judge, T. A., \& Sobral, F. (2010). Comportamento organizacional: teoria e prática no contexto brasileiro. São Paulo: Pearson.

Os conceitos apresentados na Tabela 3 apontam para a mudança de enfoque ao longo do tempo. Em meados do século passado, no centro estava o líder, o qual exercia uma postura diretiva sobre o comportamento dos liderados. De individual e centrada no líder, a liderança passou também a considerar o conjunto de pessoas envolvidas no processo de influência. Palavras como objetivos, metas e resultados, utilizadas nos conceitos, remetem à importância do alinhamento da relação com as diretrizes organizacionais.

A complexidade do fenômeno da liderança fica evidente na Tabela 4. Observa-se um aumento da abrangência, da interdependência, e a possibilidade de seu desenvolvimento. 
Tabela 4

Síntese da Evolução do Pensamento sobre Liderança

\begin{tabular}{|c|c|c|}
\hline $\begin{array}{l}\text { Nível de complexidade e } \\
\text { abrangência do conceito de } \\
\text { liderança }\end{array}$ & Definição de Liderança & Teorias ilustrativas de Liderança \\
\hline Básico & $\begin{array}{l}\text { O papel da liderança é baseado em } \\
\text { autoridade. }\end{array}$ & $\begin{array}{l}\text { Teoria dos Traços. } \\
\text { Comportamento dos Líderes. }\end{array}$ \\
\hline Intermediário & $\begin{array}{l}\text { Liderança é um processo de } \\
\text { influência entre indivíduos. } \\
\text { Os papéis desempenhados por esses } \\
\text { indivíduos são importantes no } \\
\text { exercício da influência. }\end{array}$ & $\begin{array}{l}\text { Teoria da troca entre líder e liderado } \\
(\mathrm{LMX}) \text {. }\end{array}$ \\
\hline Avançado & $\begin{array}{l}\text { Liderança é uma propriedade } \\
\text { compartilhada de um sistema social } \\
\text { que inclui interdependências entre } \\
\text { indivíduos. } \\
\text { Pode também envolver papéis e } \\
\text { processos de influência dependendo } \\
\text { da situação. }\end{array}$ & $\begin{array}{l}\text { Inclui todos os elementos abaixo: } \\
\text { Desenvolvimento de habilidades } \\
\text { individuais. } \\
\text {. Construção de relacionamentos. } \\
\text { empowerment. } \\
\text {. Colaboração. } \\
\text {. Trabalho para além das fronteiras. }\end{array}$ \\
\hline
\end{tabular}

Nota. Fonte: adaptada de Day, D. V., \& Harrison, M. M. (2007). A multilevel, identity-based approach to leadership development (p. 361). Human Resource Management Review, 17(4), 360-373. http://dx.doi.org/10.1016/j.hrmr.2007.08.007

A Tabela 4 faz alusão a algumas teorias historicamente consagradas em liderança. Sob a perspectiva da teoria de traços de personalidade, busca-se identificar qualidades e características pessoais que diferenciam líderes de não líderes; e, sob a perspectiva da teoria comportamental, buscase identificar características comportamentais que contribuam para essa mesma diferenciação. Percebese uma centralização no indivíduo (líder), uma visão prescritiva (Pereira et al., 2015) e a ênfase na relação hierárquica dos modelos tradicionais de organização. A teoria comportamental suporta a visão bidimensional de estilos de liderança (voltado às pessoas e voltado à produção), que Blake e Mouton (1964) denominaram grid gerencial (Robbins, 2005; Robbins, Judge, \& Sobral, 2010). A teoria situacional de troca entre líder e liderados, também conhecida como LMX, considera que as características das partes envolvidas passam a ser importantes, com a preocupação no autoconhecimento e o desenvolvimento mútuo (Creary, Caza, \& Roberts, 2015; Day \& Harrison, 2007; Pereira et al., 2015). Teorias sobre liderança compartilhada, coletiva e conectiva reforçam o caráter contingencial. Nesse caso, outras variáveis como processos, cultura e aspectos organizacionais aparecem, colocando a relação de liderança dependente dos indivíduos e das organizações.

Existem, também, abordagens mais recentes sobre liderança (Carvalho, Tanure, Santos, \& Lima, 2012; Dionne et al., 2014; Esper \& Cunha, 2015). Trata-se das abordagens cultural, visionária, autêntica, carismática, ética e a dicotomia transformacional - transacional, além da incorporação de outras teorias vindas da psicologia. Nessa fase, percebe-se que as teorias começam a considerar variáveis exógenas, como a política (Pereira et al., 2015), bem como ambiguidades, incertezas e gestão de significados. A abordagem carismática pode ser vista como uma retomada da abordagem dos traços, enquanto a transformacional/transacional era uma combinação do movimento comportamental com o movimento contingencial (Fonseca, Porto, \& Borges-Andrade, 2015), isto é, as abordagens evoluem incorporando novas variáveis às bases teóricas iniciais.

Uma crítica feita por Bennis e Nanus (1985) na década de 1980 - que permanece válida até a atualidade - versa sobre a operacionalização do conceito de liderança. As definições refletem modismos e tendências que não necessariamente representam a realidade ou têm relevância prática. Em resposta a críticas dessa natureza, inclusive, assistiu-se ao surgimento de trabalhos fora do ambiente acadêmico 
que se caracterizaram pelo caráter processual e prescritivo (ex: como liderar). Esses trabalhos se consagraram pela proposição de modelos, estratégias e regras (Bennis \& Nanus, 1985; Blanchard, 2007; Charan, Drotter, \& Noel, 2012; Kouzes \& Posner, 1997, Ulrich, Smallwood, \& Sweetman, 2011) que ajudassem no aprimoramento de habilidades de liderança.

Embora tenha havido avanço nos estudos sobre o líder e a liderança, sobretudo nas três últimas décadas, não se pode deixar de apontar uma demanda latente: o entendimento mais amplo sobre o tema, que passa pela investigação das relações organizacionais envolvendo o líder. Trata-se de um espaço vazio que, para ser preenchido, requer uma mudança de perspectiva - de psicológica para gestão.

\section{As relações organizacionais envolvendo o líder e a liderança: ênfase dos estudos e trabalhos}

Mesmo considerando a diversidade de conceitos, teorias e modelos desenvolvidos e utilizados para o estudo da liderança, destacando os aqui mencionados (Bergamini, 2009; Day \& Harrison, 2007; Robbins et al., 2010), pode-se dizer, que a essência do papel do líder, numa organização é influenciar e facilitar esforços individuais e coletivos para o atingimento de objetivos compartilhados (Day \& Harrison, 2007; C. M. Fernandes, Siqueira, \& Vieira, 2014; Yukl, 2012). Trata-se de um exercício que envolve recursos, relações e resultados (Blake \& McCanse, 1991). Assim, esse papel organizacional pode ser analisado sob vários aspectos: relação líder-indivíduo, relação líder-grupo, processo de influência, formas de facilitação, definição e aferição de objetivos e resultados, além de relações com outras variáveis organizacionais que atuam de forma moderadora.

Tornando a análise mais estruturada, Yukl (2012) argumenta que o comportamento do líder pode ser orientado à tarefa (clarificação, planejamento, monitoramento de atividades e solução de problemas); às pessoas (suporte, desenvolvimento, reconhecimento e empowerment - empoderamento); à mudança (defesa, visão, incentivo à inovação, facilitação do aprendizado coletivo) ou ainda ter uma orientação externa (networking, monitoramento do mercado, representação externa). Essa perspectiva expande a visão do grid da liderança que enfatiza as dimensões produção e pessoas (Blake \& McCanse, 1991). No exercício do seu papel, o líder não terá um comportamento restrito a uma das quatro dimensões mencionadas, mas ele acaba apresentando ênfases mais específicas em algumas dessas áreas, de acordo com suas características pessoais (Waldman, Balthazard, \& Peterson, 2011).

Com isso, os estudos acadêmicos que analisam o líder e a liderança abordam diferentes aspectos e estes, das mais variadas formas. Os estudos são extensos, ricos na diversidade de abordagens, mas talvez escassos no aprofundamento de algumas temáticas.

A relação do líder com indivíduos e grupos é estudada sempre tomando por base alguma teoria de liderança associada à determinada temática organizacional, como justiça, uso da comunicação, confiança ou clima organizacional, entre outros. Zhang, Lepine, Buckman e Wei (2014) verificaram que a liderança transformacional fortalece a relação entre desafios e justiça, enquanto líderes transacionais minimizam a relação entre obstáculos e justiça - entendendo a justiça como a percepção favorável dos resultados e benefícios recebidos por um empregado na troca pelas suas contribuições. Campos, Araújo, Moraes e Kilimnik (2013) observaram uma correlação positiva entre o estilo de liderança participativoconsultivo e a confiança organizacional (vulnerabilidade de uma parte em relação à ação de outra parte, reforçada por expectativas positivas). Vries, Bakker-Pieper e Oostenveld (2010) concluíram que líderes carismáticos e aqueles orientados às pessoas têm melhor repertório de comunicação do que outros orientados às tarefas, além de o exercício da comunicação garantir melhores resultados.

Quando se relaciona liderança a resultados, estes são entendidos como atitudes dos liderados ou impacto da execução da tarefa/atividade e não a resultados organizacionais. Alguns trabalhos chegam a conclusões interessantes, demonstrando relações com o ambiente organizacional e com o perfil do líder pesquisado. Martin, Liao e Campbell (2013) aferem que o líder empoderador tem influência sobre o resultado do trabalho e sobre a atitude proativa de seus subordinados. Já o líder diretivo influencia somente o resultado do trabalho. Porém, quando os liderados estão satisfeitos com o líder, a influência na atitude proativa também é verificada no estilo diretivo. Dois trabalhos que estudam a relação com o 
comprometimento aferiram resultados com direções praticamente opostas. Maciel e Nascimento (2013) construíram três clusters de liderança transformacional e verificaram a relação desses três clusters com as três dimensões do comprometimento - afetiva, normativa e instrumental (continuidade). A relação com a dimensão instrumental é mediada pelo grau de instrução dos liderados. Já, o estudo de C. M. Fernandes, Siqueira e Vieira (2014), utilizando o conceito de estilos de liderança (adaptado de Lewin e Lippett e de White e Goleman), verificou que o repertório de estilos utilizados tem um papel moderador fraco entre suporte organizacional e comprometimento afetivo. Dias e Borges (2015) verificaram que, no setor público, o estilo transacional está mais associado ao melhor desempenho da equipe por atrelar desempenho à recompensa. Ainda, Chami-Malaeb e Garavan (2013) propuseram, como resultado de pesquisa bibliográfica, um modelo conceitual que relaciona o desenvolvimento de práticas de liderança com o comprometimento afetivo e a intenção em permanecer na organização, entre outros aspectos.

Os estudos mencionados não esgotam os estudos recentes sobre líderes, liderança e suas relações com indivíduos e resultados, mas demonstram vasta gama de possibilidades, abordagens e diferentes achados. Vale ressaltar que todos esses estudos são de abordagem quantitativa.

Quando a relação muda para líder e tarefa ou processo, numa tentativa de colocar o líder como um ator importante na definição e na implementação de estratégias, considerando, inclusive, seu espaço organizacional (O'Reilly, Caldwell, Chatman, Lapiz, \& Self, 2010), os estudos se tornam mais escassos. A pesquisa sobre as relações líder e estratégia, líder e execução retorna poucos resultados, e estes, em quase sua totalidade, são discussões conceituais que posicionam o líder como o elo entre missão, visão, valores, estratégias, objetivos, metas e pessoas (ex: C. R. Fernandes \& Silva, 2015; Galpin \& Whittington, 2012; Maureen, 2008) nos mais variados contextos, relacionando, também, outras temáticas como sustentabilidade, qualidade, entre outras. Rowe (2002) tentou desenvolver o conceito de líder estratégico como um elemento de criação de valor na organização. Seu trabalho define características desse líder como uma mistura ou equilíbrio entre as atuações e as posturas visionárias e gestoras, mais ou menos como argumenta Peter Drucker - os melhores líderes são, primeiro e antes de mais nada, gerentes eficazes (Collins, 2012). Os estudos de Shaap (2006) e de Maritz, Pretorius e Plant (2011) parecem ser dos poucos trabalhos empíricos que investigam a relação do líder com a estratégia e com o seu papel organizacional. Infelizmente, os achados do primeiro estudo não foram conclusivos com relação à atuação do líder e a eficácia da implementação estratégica, e os do segundo mais enfatizam a utilização mista da estratégia emergente e da estratégia deliberada, sem uma diferenciação específica da atuação do líder.

Três trabalhos detalham a ênfase da produção acadêmica sobre liderança, no Brasil: Delfino, Silva e Rohde (2010), Santos et al. (2013) e Fonseca, Porto e Borges-Andrade (2015). O primeiro trabalho retrata que, entre 1996 e 2009, a ênfase dos estudos foi sobre: constructos de liderança, modelos, perfil, estilos de liderança e relação líder-liderado. O segundo trabalho, observando o período de 2007 a 2012, reforça a mesma ênfase, acrescentando análises de diferenças demográficas nos líderes e de processos de desenvolvimento de liderança. O terceiro trabalho, por sua vez, ressalta que os estudos nacionais ainda são muito incipientes quando comparados a outras áreas do comportamento organizacional e aos estudos realizados fora do Brasil. Percebe-se, assim, que, apesar de abundante, diverso e complexo, o estudo e a pesquisa sobre a liderança circundam o líder, suas interações/relações com as pessoas e alguns impactos organizacionais, mas pouco articulam as quatro dimensões mencionadas por Yukl (2012), que posicionaria o líder como elo fundamental no alinhamento organizacional. Isso se torna um gap e uma oportunidade de avanço nos estudos sobre o tema.

\section{O papel do líder na gestão de pessoas}

Sob a perspectiva da gestão de pessoas, um dos assuntos que tem sido amplamente investigado é o desenvolvimento da liderança (Avolio, Avey, \& Quisenberry, 2010; Boyce, Zaccaro, \& Wisecarver, 2010; Day, Fleenor, Atwater, Sturn, \& McKee, 2014; Freitas, Klein, \& Fargundes, 2015; Ladegard \& Gjerde, 2014; O'Connell, 2014; Riggio \& Mumford, 2011; Solansky, 2010). Partindo-se da visão de que a liderança pode ser desenvolvida (Blake \& McCanse, 1991), a maioria dos estudos acadêmicos enfatizam o desenvolvimento da liderança como um dos produtos ou resultados da gestão de pessoas. $\mathrm{O}$ 
desenvolvimento é estudado com foco individual, grupal ou organizacional. Mais recentemente, a ênfase no líder global passou a fazer parte do foco organizacional.

Sob o foco individual, os estudos enfatizam a personalidade, a identidade, as habilidades, o autodesenvolvimento, o aprendizado por meio de experiências, processos de coaching e mentoring, qualidade de vida, competências emocionais, entre outros temas. Quando refletindo o grupo, a ênfase está no networking, no desenvolvimento de grupos e equipes e em ferramentas de avaliação multidirecional. O desenvolvimento organizacional sempre reforça competências gerenciais e processos de mudança. A abordagem global reflete, na maioria das vezes, a definição de um perfil de liderança global, treinamentos interculturais e desenvolvimento da experiência internacional. Além dos trabalhos já citados, dois trabalhos, em especial, fazem uma revisão importante sobre a evolução da literatura e da pesquisa sobre desenvolvimento de liderança: Day (2001) e Day, Fleenor, Atwater, Sturn e McKee (2014). Vale ressaltar, também, que a linha de estudos que se ocupa da temática desenvolvimento do líder ou da liderança ainda tem foco muito centrado no indivíduo, seu autoconhecimento, aprimoramento e evolução, e não no papel de elo organizacional como, por exemplo, gestor das pessoas, parecendo que a evolução das teorias não se reflete nos objetivos dos estudos empíricos. Uma diferença, nesse sentido, é o trabalho de Reichard e Johnson (2001), que busca, com base em um modelo conceitual proposto, articular estratégia organizacional, processos de recursos humanos e liderança, mas a ênfase, ainda, é o desenvolvimento de lideranças.

Estudiosos de gestão de pessoas discutem a gestão estratégica, o alinhamento com a estratégia organizacional (Baron \& Kreps, 1999; Becker, Huselid, \& Ulrich, 2001; Legge, 2005; Schuler \& Jackson, 1987; Silveira, 2014; Ulrich \& Brockbank, 2005), as políticas e as práticas de gestão (Baron \& Kreps, 1999; Lepak \& Snell, 2002; Schuler \& Jackson, 1987; Veloso et al., 2014) e os papéis organizacionais (Baron \& Kreps, 1999; Lawler, 2005; Ulrich, 1997, 2004). Na discussão sobre papéis, a visão de que o processo de gestão deve ser de toda a organização (Bianchi, 2008), de que os profissionais de recursos humanos devem ser parceiros estratégicos, e que os gestores são os responsáveis pela gestão de pessoas está sempre presente como fundamento teórico, mas é pouco estudada empiricamente. O líder, então, aparece como um produto ou resultado da gestão de pessoas e não com um dos atores desse processo.

Ulrich $(1997,2004)$ trabalhou em detalhe os papéis na gestão de pessoas: gestão dos recursos estratégicos, gestão da transformação e mudança, gestão da contribuição das pessoas e gestão da infraestrutura. Este último papel é atribuído prioritariamente a profissionais de recursos humanos, mas os demais devem ser compartilhados com os gestores/líderes e também profissionais das organizações. Segundo o autor, o papel de gestor da contribuição das pessoas - aumento do comprometimento e da competência individual das pessoas é, essencialmente, um papel do líder. Infelizmente, o estudo empírico do exercício desse papel está endereçado aos profissionais de $\mathrm{RH}$, a exemplo dos trabalhos de Bianchi (2008) e de Moreira (2010). A proposta de Ulrich pode não ser perfeita, mas é um ponto de partida para ampliar o campo de estudo sobre o papel organizacional do líder como elo na gestão de pessoas.

Observado que o estudo da gestão de pessoas, embasado pelas perspectivas, segue uma linha mais processual, isto é, fortemente embasado por filosofias, práticas e articulações entre estas e que o estudo da liderança pouco vislumbra o líder como um gestor de processos, em especial o processo de gestão de pessoas, faz-se necessária uma amarração entre esses elos para que a gestão de pessoas seja vislumbrada de forma prática e efetiva.

\section{As Três Dimensões na Gestão de Pessoas: Estratégia de Negócios, Práticas de Gestão e o Papel do Líder na Execução dessas Práticas}

Como já discutida, a gestão das pessoas não é um processo compartimentado. Se, por um lado, políticas e práticas, isoladamente, não bastam para assegurar a gestão, por outro, somente a influência 
exercida pelo líder sobre os indivíduos também não. Apenas a combinação de políticas e práticas estabelecidas, compartilhadas e implementadas por um líder atuante é capaz de direcionar o comportamento das pessoas promovendo mensagens articuladas e coletivas, sempre com foco nos indivíduos e conectadas com o objetivo da organização.

Diante disso, Gratton e Truss (2003), com um estudo em sete organizações ao longo de 10 anos, desenvolveram um modelo que chamaram de estratégia tridimensional de pessoas. Esse modelo incorpora três dimensões: alinhamento vertical entre estratégia de negócio e estratégia de gestão de pessoas; alinhamento horizontal entre as políticas e práticas de gestão de pessoas; e um terceiro eixo, que verifica em que grau as políticas e práticas são colocadas em prática (perspectiva da percepção das pessoas sobre a atuação do líder e dos profissionais de recursos humanos). O modelo utiliza os conceitos de contingência e configuração (Delery \& Doty, 1996; Martín-Alcázar et al., 2005) e incorpora dois atores: os empregados e suas experiências com as práticas de gestão de pessoas; e os líderes, com seus comportamentos e valores na implementação dessas práticas. Ao incorporar esses dois atores e ainda discutir o papel dos profissionais de recursos humanos, as autoras parecem debater, de forma não declarada, a multiplicidade de papéis apresentada por Ulrich (1997). Gratton e Truss (2003) buscam o foco da implementação na gestão de pessoas, evoluindo na integração entre práticas de gestão e o exercício dessas práticas pela ação dos líderes e suas relações com os liderados.

Por se tratar de uma visão tridimensional, o modelo parece ser de análise complexa, embora cada dimensão seja aparentemente simples: alinhamento vertical, alinhamento horizontal e papéis organizacionais na gestão de pessoas. Resulta em uma combinação de 8 padrões situacionais relacionando a estratégia organizacional, as práticas de gestão de pessoas, a ação e o discurso do líder, que traduz o momento de uma organização de forma estática e tipificada. Não se pode esquecer que este é o objetivo de um modelo: demonstrar uma posição que sugira uma análise para uma ação organizacional.

A utilização combinada do modelo de Gratton e Truss (2003) com o modelo de papéis de Ulrich (1997), isto é, integrar o alinhamento entre estratégia organizacional e as políticas e práticas de gestão de pessoas, o alinhamento entre as práticas de gestão e o exercício dos papéis da liderança na gestão de pessoas (gestor de recursos estratégicos, gestor da transformação e mudança e gestor da contribuição das pessoas) pode contribuir para a evolução dos estudos no tema para uma visão mais voltada à implementação na gestão de pessoas, com parâmetros bem específicos.

Mesmo utilizando modelos preconcebidos, o que tornaria a investigação de certa forma universalizada, essa abordagem enfatizaria que estratégias e práticas são fundamentais, mas não se auto implementam, e que a mediação do líder é necessária para sustentar o alinhamento entre estratégia organizacional, práticas de gestão e comportamento das pessoas. A gestão das pessoas ficaria enfatizada como uma ação executada de forma contínua e, ainda, o estudo do papel do líder ganharia o enfoque multidimensional, como sustenta Yukl (2012),

O uso integrado desses modelos para investigação empírica, que pode, ainda, levar em consideração o espaço organizacional de O'Reilly, Caldwell, Chatman, Lapiz e Self (2010) elevaria o estudo da gestão de pessoas para outro patamar - o da implementação estratégica, levando em conta atores e processos organizacionais.

\section{Reflexões e Considerações}

A discussão sobre gestão estratégica de pessoas é, de certa forma, recente, e pauta-se na articulação de funções, na diferenciação de recursos e no alinhamento de comportamentos (como sustentam suas fundamentações teóricas). Também não faltam perspectivas teóricas que suportem estudos empíricos sobre políticas e práticas. Se, por um lado, as fundamentações teóricas sustentam aspectos relacionais múltiplos (ambiente, processos e pessoas), por outro, as perspectivas estão mais 
centradas nas relações entre os processos (estratégias, políticas e práticas). Essa constatação sugere uma lacuna de estudo relacionada à implementação da gestão de pessoas.

A evolução do estudo sobre o papel da área de recursos humanos não supre essa lacuna, porque continua centrada na formulação das práticas e ainda coloca o profissional de recursos humanos com um papel consultivo na organização. Para discutir a efetividade da gestão ou das práticas de gestão, outros atores organizacionais precisam ser incluídos, como os líderes, que exercem um papel importante de mediação no processo, e os profissionais que precisam compreender objetivos e processos para direcionar seu próprio comportamento. O líder, além de elo na gestão, pode, também, servir como um filtro entre a intenção da gestão de pessoas (conjunto arquitetado de políticas e práticas) e a percepção dessas práticas pelos profissionais em uma organização (baseada na experiência resultante da utilização das práticas).

Apesar de a liderança ser uma temática mais antiga de estudo, quando comparada à gestão estratégica de pessoas, a ênfase, apesar da evolução do campo teórico, está no indivíduo que atua como líder e não no papel exercido por este indivíduo, na organização, considerando outras variáveis contingenciais. A atuação como gestor de pessoas - aquele que utiliza o conjunto de políticas e práticas para mediar sua relação com o liderado - parece ainda ser pouco explorada. Esse aspecto torna fragmentado tanto o estudo de gestão estratégica de pessoas quanto o estudo sobre o líder e sua atuação, revelando um retrato vago e ainda impreciso sobre esse fenômeno.

O aprofundamento da discussão sobre implementação da gestão estratégica de pessoas precisa incorporar e ampliar a compreensão sobre o papel do líder. As quatro orientações (tarefa, pessoas, mudança organizacional e orientação externa) do líder propostas por Yukl (2012), as definições de espaço organizacional de O'Reilly et al. (2010), bem como o modelo tridimensional (estratégia, práticas e exercício de papéis) de Gratton e Truss (2003), e o modelo de papéis na gestão de pessoas de Ulrich (1997) podem ser utilizados de forma integrada para iniciar um outro momento no estudo da gestão estratégica de pessoas. Não se trataria, no início, de inovação teórica, mas sim de uma inovação metodológica que poderia suscitar novas possibilidades sobre questões relacionadas à implementação estratégica na gestão de pessoas. O teste dessa integração de conceitos e modelos pode resultar em uma inovação teórica, levando-se em conta que os conceitos sugeridos também não são tão contemporâneos.

Enfatizando o exercício dos papéis do líder, estudos qualitativos podem aprofundar a análise sobre as características dos diversos papéis sob a perspectiva do negócio, tomando-se, inclusive, como pano de fundo, os estudos de Carvalho, Tanure, Santos e Lima (2012), que verificam divergências das características dos líderes brasileiros com as do modelo transformacional (base para os modelos de Ulrich, 1997; Yukl, 2012).

O papel de mediação do líder na gestão de pessoas (seja na elaboração ou na utilização das práticas de gestão, ou, ainda, na forma de relacionamento com o liderado) pode ser verificado por meio de análises quantitativas. Outras variáveis contingenciais, como estratégia e comprometimento, ainda podem ser incorporadas. A preocupação com a implementação da gestão estratégica de pessoas não pode perder de vista a perspectiva do resultado organizacional. Ao contrário, ela deve sustentar a relevância do resultado, evidenciando como se chegar a este.

Outras temáticas como cultura organizacional, estrutura organizacional, comunicação, ambiente de trabalho e suporte organizacional para o efetivo exercício do papel do líder podem, também, ser gradativamente incorporadas aos estudos, evidenciando a complexidade do processo de gestão de pessoas.

Um maior alinhamento entre questões como atuação do líder, liderança e gestão estratégica de pessoas contribui tanto com a academia - na continuidade, desenvolvimento e aprofundamento do campo de estudo intitulado Implementação Estratégica de Pessoas quanto com o mundo corporativo, tratando de um dilema de enorme relevância prática: qual é a contribuição do líder na gestão de pessoas e como garantir essa contribuição? 


\section{Nota}

${ }^{1}$ Parte do projeto Gestão Estratégica de Pessoas e Comprometimento Organizacional: um estudo integrador, financiado pelo CNPq (45726920146) em vigor a partir de novembro/2014.

\section{Referências}

Albuquerque, L. G. (2002). A gestão estratégica de pessoas. In M. T. Fleury (Org.), As pessoas na organização (pp. 35-50). São Paulo: Editora Gente.

Alfes, K., Truss, C., Soane, E. C., Rees, C., \& Gatenby, M. (2013). The relationship between line manager behavior, perceived HRM practices, and individual performance: examining the mediating role of engagement. Human Resource Management, 52(6), 839-859. http://dx.doi.org/10.1002/hrm.21512

Armstrong, M. (2011). Armstrong's handbook of human resource management. London: Kogan Page.

Arthur, J. B. (1992). The link between business strategy and industrial relations systems in American steel minimills. ILR Review, 45(3), 488-506. http://dx.doi.org/10.1177/001979399204500306.

Avolio, B. J., Avey, J. B., \& Quisenberry, D. (2010). Estimating return on leadership development investment. The Leadership Quarterly, 21(1), 633-644. http://dx.doi.org/10.1016/j.leaqua.2010.06.006

Baron, J. N., \& Kreps, D. M. (1999). Strategic human resources: framework for general managers. Hoboken: John Wiley \& Sons, Inc.

Bassi, L., \& McMurrer, D. (2007). Maximizing your return on people. Harvard Business Review, 85(3), $115-123$.

Batt, R. (2002). Managing customer services: human resource practices, quit rates, and sales growth. Academy of Management Journal, 45(3), 587-597. http://dx.doi.org/10.2307/3069383.

Becker, B. E., Huselid, M. A., \& Ulrich, D. (2001). Gestão estratégica de pessoas com scorecard. Rio de Janeiro: Campus.

Bennis, W., \& Nanus, B. (1985). Leaders: the strategies for taking charge. New York: Harper \& Row.

Bergamini, C. W. (2009). Liderança: administração do sentido. São Paulo: Atlas.

Bianchi, E. M. P. G. (2008). Alinhando estratégia de negócio e gestão de pessoas para obtenção de vantagem competitiva (Dissertação de mestrado). Faculdade de Economia, Administração e Contabilidade, Universidade de São Paulo, São Paulo, Brasil.

Blake, R., \& McCanse, A. A. (1991). Leadership dilemmas-grid solutions. Austin: Gulf Publishing Company.

Blake, R., \& Mouton, J. (1964). The managerial grid: the key to leadership excellence. Houston: Gulf Publishing Co.

Blanchard, K. (2007). Liderança de alto nível: como criar e liderar organizações de alto desempenho. Porto Alegre: Bookman.

Bos-Nehles, A. C., Riemsdijk, M. J. van, \& Looise, J. K. (2013). Employee perceptions of line management performance: applying the AMO Theory to explain the effectiveness of Line 
Managers' HRM implementation. Human Resource Management, 52(6), 861-877. http://dx.doi.org/10.1002/hrm.21578

Boyce, L. A., Zaccaro, S. J., \& Wisencarver, M. Z. (2010). Propensity of self-development of leadership attributes: understanding, predicting, and supporting performance of leader self-development. The Leadership Quarterly, 21(1), 159-178. http://dx.doi.org/10.1016/j.leaqua.2009.10.012

Boxall, P., \& Purcell, J. (2003). Strategy and human resource management. Basingstoke: Palgrave Macmillan.

Campos, H. L., Araújo, E. M., Moraes, L. F. R., \& Kilimnik, Z. M. (2013). Estilos de liderança e interferência no nível de confiança organizacional: um estudo de caso. Revista Gestão e Planejamento, 14(3), 389-409.

Carvalho, A. M., Neto, Tanure, B., Santos, C. M. M., \& Lima, G. S. (2012). Executivos brasileiros: na contramão do perfil deificado da liderança transformacional. Revista de Ciências da Administração, 14(32), 35-49. http://dx.doi.org/10.5007/2175-8077.2012v14n32p35

Chami-Malaeb, R., \& Garavan, T. (2013). Talent and leadership development practices as drivers of intention to stay in Lebanese organisations: the mediating role of affective commitment. The International Journal of Human Resource Management, 24(21), 4046-4062. http://dx.doi.org/10.1080/09585192.2013.789445

Charan, R., Drotter, S., \& Noel, J. (2012). Pipeline de liderança: o desenvolvimento de líderes como diferencial competitivo. São Paulo: Elsevier.

Collins, J. C. (2012). Effective management - the best leaders first excel at this task. Leadership Excellence, 29(4), 3-4.

Collins, J. C., \& Porras, J. I. (1997). Built to last: successful habits of visionary companies. New York: Harper Collis Publishers.

Creary, S. J., Caza, B. B., \& Roberts, L. M. (2015). Out of the box? How managing a subordinate's multiple identities affects the quality of a manager-subordinate's relationship. Academy of Management Review, 40(4), 538-562. http://dx.doi.org/10.5465/amr.2013.0101

Datta, D. K., Guthrie, J. P., \& Wright, P. M. (2005). Human resource management and labor productivity. Does industry matter? Academy of Management Journal, 48(1), 135-145. http://dx.doi.org/10.5465/AMJ.2005.15993158

Day, D. V. (2001). Leadership Development: a review in context. The Leadership Quarterly, 11(4), 581-613. http://dx.doi.org/10.1016/s1048-9843(00)00061-8

Day, D. V., Fleenor, J. W., Atwater, L. E., Sturn, R. E., \& McKee, R. A. (2014). Advances in leader and leadership development: a review of 25 years of research and theory. The Leadership Quarterly, 25(1), 63-82. http://dx.doi.org/10.1016/j.leaqua.2013.11.004

Day, D. V., \& Harrison, M. M. (2007). A multilevel, identity-based approach to leadership development. Human Resource Management Review, 17(4), 360-373. http://dx.doi.org/10.1016/j.hrmr.2007.08.007

Delaney, J. J., \& Huselid, M. A. (1996). The impact of human resource management practices on perceptions of organizational performance. Academy of Management Journal, 39(4), 949-969. http://dx.doi.org/10.2307/256718

Delery, J. E., \& Doty, D. H. (1996). Modes of theorizing in strategic human resource management: tests of universalistic, contingency and configurational performance predictions. Academy of Management Journal, 39(4), 802-835. http://dx.doi.org/10.2307/256713 
Delfino, I. A. de L., Silva, A. B. da, \& Rohde, L. R. (2010, setembro). A produção acadêmica sobre liderança no brasil: uma análise bibliométrica dos artigos publicados em eventos e periódicos entre 1995 e 2009. Anais do Encontro da Associação Nacional de Pós-Graduação e Pesquisa em Administração, Rio de Janeiro, RJ, Brasil, 34.

Demo, G., Neiva, E. R., Nunes, I., \& Rozzett, K. (2012). Human resources management policies and practices scale (HRMPPS): exploratory and confirmatory factor analysis. Brazilian Administration Review, 9(4), 395-420. Recuperado de http://www.scielo.br/pdf/bar/v9n4/aop0512.pdf.

Dias, M. A. M. J., \& Borges, R. S. G. (2015). Estilos de liderança e desempenho de equipes no setor público. Revista Eletrônica de Administração, 80(1), 200-221. Recuperado de http://www.scielo.br/pdf/read/v21n1/1413-2311-read-21-01-00200.pdf. http://dx.doi.org/10.1590/1413-2311.0542014.53468

Dionne, S. D., Gupta, A., Sotak, K. L., Shirreffs, K. A., Serban, A., Hao, C., Kim, D. H. \& Yammarino, F. J. (2014). A 25-year perspective on level of analysis in leadership research. The Leadership Quarterly, 25(1), 6-35. http://dx.doi.org/10.1016/j.leaqua.2013.11.002

Esper, A. J. F., \& Cunha, C. J. C. A. (2015). Liderança autêntica: uma revisão integrativa. NAVUS. Revista de Gestão e Tecnologia, 5(2), 60-72.

Fernandes, C. M., Siqueira, M. M. M., \& Vieira, A. M. (2014). Impacto da percepção de suporte organizacional afetivo: o papel moderador da liderança. Revista Pensamento Contemporâneo em Administração, 8(4), 140-162. http://dx.doi.org/10.12712/rpca.v8i4.396

Fernandes, C. R., \& Silva, A. B. da (2015). Integration of strategy and leadership: an introduction. Revista Ibero-Americana de Estratégia, 14(4), 63-77. http://dx.doi.org/10.5585/ijsm.v14i4.2215

Fischer, A. L. (2002). Um resgate conceitual e histórico dos modelos de gestão de pessoas. In M. T. Fleury (Coord.), As pessoas na organização (pp. 11-34). São Paulo: Editora Gente.

Flickinger, M., Allscher, M., \& Fiedler, M. (2016). The mediating role of a leader-member exchange: a study of job satisfaction and turnover intentions in temporary work. Human Resource Management Journal, 26(1), 46-62. http://dx.doi.org/10.1111/1748-8583.12091.

Fonseca, A. M., Porto, J. B., \& Borges-Andrade, J. E. (2015). Liderança: um retrato da produção cientifica brasileira. Revista de Administração Contemporânea, 19(3), 290-310. Recuperado de $\mathrm{http} / / / \mathrm{www} . \mathrm{scielo.br/pdf/rac/v19n3/1415-6555-rac-19-03-00290.pdf.}$ http://dx.doi.org/10.1590/1982-7849rac20151404

Foroni, P. G. (2014). Gestão estratégica de pessoas: estudo de caso sobre o alinhamento (Dissertação de mestrado). Universidade de São Paulo, São Paulo, SP, Brasil.

Freitas, J. C. da S., Jr., Klein, A. Z., \& Fargundes, P. M. (2015). Uma proposta de instrumento quantiqualitativo para autoavaliação de competências da liderança. Revista de Administração da UNIMEP, 13(3), 1-27. http://dx.doi.org/10.15600/1679-5350/rauv13n3p1-27

Galpin, T., \& Whittington, J. L. (2012). Sustainability leadership: from strategies to results. Journal of Business Strategy, 33(4), 40-48. http://dx.doi.org/10.1108/02756661211242690

Gratton, L., \& Truss, C. (2003). The three-dimensional people strategy: putting human resources policies into action. Academy af Management Executive, 17(3), 74-87. http://dx.doi.org/10.5465/AME.2003.10954760

Hrebiniak, L. G. (2006). Fazendo a estratégia funcionar: o caminho para uma execução bem-sucedida. Porto Alegre: Bookman. 
Huselid, M. A. (1995). The impact of human resource management practices on turnover, productivity, and corporate financial performance. Academy of Management Journal, 38(3), 635-670. http://dx.doi.org/10.2307/256741

Jamrog, J. J., \& Overholt, M. H. (2004). Building a strategic HR function: continuing the evolution. Human Resource Planning, 27(1), 51-62.

Jiang, K., Lepak, D. P., Han, K., Hong, Y., Kim, A., \& Winler, A. (2012). Clarifying the construct of human resource systems: relating human resource management to employee performance. Human Resource Management Review, 22(2), 73-85. http://dx.doi.org/10.1016/j.hrmr.2011.11.005

Kelliher, C., Hailey, V. H., \& Farndalde, E. (2013). Employee engagement in multinational organizations. In C. Truss, R. Delbridge, K. Alfes, A. Shantz, \& E. Soane (Orgs.), Employee engagement in theory and practice (pp. 180-194). New York: Routledge.

Kouzes, J. M., \& Posner, B. Z. (1997). O desafio da liderança. Rio de Janeiro: Campus.

Ladegard, G., \& Gjerde, S. (2014). Leadership coaching, leader role-efficacy, and trust in subordinates: a mixed methods study assessing leadership coaching as a leadership development tool. The Leadership Quarterly, 25(4), 631-646. http://dx.doi.org/10.1016/j.leaqua.2014.02.002

Lakshman, C, (2014). Leveraging human capital through performance management process: the role of leadership in the USA, France and India. The International Journal of Human Resource Management, 25(10), 1351-1372. http://dx.doi.org/10.1080/09585192.2013.870310

Lawler, E. E., III (2005). Make strategic partnership a reality. Strategic HR Review, 4(3), 3. http://dx.doi.org/10.1108/14754390580000595

Lee, M. A. (1994). Matching human resource strategy to organizational strategy: the behavioral perspective revisited (Dissertation). School of Business and Entrepreneurship Nova Southeast, University of Canada, Canada.

Legge, K (2005). Human resource management: rhetorics and realities. New York: Palgrave.

Lepak, D. P., \& Snell, S. A. (1999). The human resource architecture: toward a theory of human capital allocation and development. Academy of Management Review, 24(1), 31-48. http://dx.doi.org/10.5465/AMR.1999.1580439

Lepak, D. P., \& Snell, S. A. (2002). Examining the human resource architecture: the relationships among human capital, employment and human resources configurations. Journal of Management, 28(4), 517-543. http://dx.doi.org/10.1177/014920630202800403

Liu, D., Gong, Y., Zhou, J., \& Huang, F-C. (in press). Human resource systems, employee creativity, and firm creativity: the moderating role of the firm ownership. Academy of Management Journal. http://dx.doi.org/10.5465/amj.2015.0230

Maciel, C. de O., \& Nascimento, M. R. (2013). Em busca de uma abordagem não atomizada para o exame das relações entre liderança transformacional e comprometimento organizacional. Revista de Administração da USP, 48(3), 544-559. http://dx.doi.org/10.5700/rausp1105

Maritz, R., Pretorious, M., \& Plant, K. (2011). Exploring the interface between strategy-making and responsible leadership. Journal of Business Ethics, 98(Suppl. 1), 101-113. http://dx.doi.org/10.1007/s10551-011-1024-5

Martín-Alcázar, F., Romero-Fernández, P. M., \& Sánchez-Gardey, G. (2005). Strategic human resource management: integrating the universalistic, contigent, configurational and contextual perspectives. The International Journal of Human Resource Management, 16(5), 633-659. http://dx.doi.org/10.1080/09585190500082519 
Martin, S. L., Liao H., \& Campbell, E. M. (2013). Directive versus empowering leadership: a field experiment comparing impacts on task proficiency and proactivity. Academy of Management Journal, 56(5), 1372-1395. http://dx.doi.org/10.5465/amj.2011.0113

Maureen, B. (2008). Leadership's role in execution. Healthcare Executive, 23(2), 66-70.

Maximiano, A. C. A. (2004). Teoria geral da administração. São Paulo: Atlas.

Miles, R. E., \& Snow, C. C. (1984). Designing strategic human resources systems. Organizational Dynamics, 13(1), 36-52. http://dx.doi.org/10.1016/0090-2616(84)90030-5

Moreira, M. M. M. (2010). Alinhamento entre gestão de pessoas e estratégia organizacional: um estudo de caso de uma empresa pública brasileira (Dissertação de mestrado). Instituto Universitário de Lisboa, Portugal.

Motta, F. C. P., \& Vasconcelos, I. F. G. de (2004). Teoria geral da administração. São Paulo: Thomson Learning.

O'Connell, P. K. (2014). A simplified framework for $21^{\text {st }}$ leader development. The Leadership Quarterly, 25(2), 183-203. http://dx.doi.org/10.1016/j.leaqua.2013.06.001

O’Reilly, C. A., Caldwell, D. F., Chatman, J. A., Lapiz, M., \& Self, W. (2010). How leadership matters: the effect of leader's alignment on strategy implementation. The Leadership Quarterly, 21(1), 104-113. http://dx.doi.org/10.1016/j.leaqua.2009.10.008

Ostroff, C., \& Bowen, D. E. (2016). Reflections on the 2014 decade award: is there strength in the construct of HR system strength? Academy of Management Review, 24(2), 196-214. http://dx.doi.org/10.5465/amr.2015.0323

Pereira, J. J., Albuquerque Maranhão, C. M. S. de, Rezende, A. F., \& Mendonça, M. C. A. (2015, setembro). Um estudo sobre a teoria tradicional de liderança: contribuições da teoria crítica. Anais do Encontro da Associação Nacional de Pós-Graduação e Pesquisa em Administração, Belo Horizonte, MG, Brasil, 39.

Pfeffer, J. (1994). Competitive advantage through people: unleashing the power of the work force. Boston: HBS Press.

Pfeffer, J. (1998). The human equation: building profits by putting people first. Boston: HBS Press.

Reichard, R. J., \& Johnson, S. K. (2011). Leader self-development as organizational strategy. The Leadership Quarterly, 22(1), 33-42. http://dx.doi.org/10.1016/j.leaqua.2010.12.005

Riggio, R. E., \& Mumford, M. D. (2011). Introduction to special issue: longitudinal studies of leadership development. The Leadership Quarterly, 22(3), 453-456. http://dx.doi.org/10.1016/j.leaqua.2011.04.002

Robbins, S. P. (2005). Comportamento organizacional. São Paulo: Pearson Prentice Hall.

Robbins, S. P., Judge, T. A., \& Sobral, F. (2010). Comportamento organizacional: teoria e prática no contexto brasileiro. São Paulo: Pearson.

Rowe, W. G. (2002). Liderança estratégica e a criação de valor. Revista de Administração de Empresas, 42(1), 7-9. http://dx.doi.org/10.1590/s0034-75902002000100003

Saá-Pérez, P., \& García-Falcón, J. M. (2011). A resource-based view of human resource management: an organizational capabilities development. The International Journal of Human Resource Management, 13(1), 123-140. http://dx.doi.org/10.1080/09585190110092848 
Santos, N. C. dos, Machado, L. A., Francischetti, C. E., Padoveze, C. L., Ferreira, L. de O., \& Oswaldo, Y. C. (2013, setembro). A relevância da liderança no Brasil: um estudo bibliométrico sobre a produção acadêmica no período de 2007 a 2012. Anais do Encontro da Associação Nacional de Pós-Graduação e Pesquisa em Administração, Rio de Janeiro, RJ, Brasil, 36.

Schuler, R. S., \& Jackson, S. E. (1987). Linking competitive strategies with human resource. Academy of Management Executive, 1(3), 207-219. http://dx.doi.org/10.5465/AME.1987.4275740

Shaap, J. I. (2006). Toward strategy implementation success: an empirical study of the role of the seniorlevel leaders in the Nevada Gaming Industry. UNLV Gaming Research \& Review Journal, 10(2), 13-37.

Silveira, V. N. S. (2014). Alinhamento estratégico e gestão estratégica de pessoas: análise conceitual e $\begin{array}{llll}\text { perspectivas teóricas. } & \text { PRETEXTO, 15(4), } & 114-133 .\end{array}$ http://dx.doi.org/10.21714/pretexto.v15i4.2769

Solansky, S. T. (2010). The evaluation of two key leadership development program components: leadership skills assessment and leadership mentoring. The Leadership Quarterly, 21(4), 675681. http://dx.doi.org/10.1016/j.leaqua.2010.06.009

Spisak, B. R., O’Brien, M. J., Ncholson, N., \& Van Vugt, M. (2015). Niche cosntruction and the evolution of leadership. Academy of Management Review, 40(2), 291-306. http://dx.doi.org/10.5465/amr.2013.0157

Thompson, A. A., \& Strickland, A. J., III (1996). Strategic management: concepts and cases. Boston: McGraw-Hill.

Tichy, N., Fombrun, C., \& Devanna, M. (1982). Strategic human resource management. Sloan Management Review, 23(2), 47-61.

Ulrich, D. (1997). Human resource champions. Boston: HBSP.

Ulrich, D. (Org.). (2004). Recursos humanos estratégicos: novas perspectivas para os profissionais de $R H$. São Paulo: Futura.

Ulrich, D., \& Brockbanck, W. (2005). The HR value proposition. Boston: HBSP.

Ulrich, D., Smallwood, N., \& Sweetman, K. (2011). O código da liderança: cinco regras para fazer diferença. Rio de Janeiro: Best Seller.

Veloso, E. F. R., Trevisan, L. N., Amorin, W. A. C. de, Silva, R. C. da, Fischer, A. L., \& Dutra, J. S. (2014). Brazilian human resources in a polarized Latin America. InternexT Revista Eletrônica de Negócios Internacionais, 9(3), 1-15. Recuperado de http://internext.espm.br/index.php/internext/article/download/210/204

Ventura, V. L. de S., \& Leite, N. R. P. (2014). Percepção da influência da gestão estratégica de pessoas no comprometimento organizacional. PRETEXTO, 15(3), 11-28. http://dx.doi.org/10.21714/pretexto.v15i3.1447

Vries, R. E. de, Bakker-Pieper, A., \& Oostenveld, W. (2010). Leadership = Communication? The relations of leaders' communication styles with leadership styles, knowledge sharing and leadership outcomes. Journal of Business and Psychology, 25(3), 367-380. http://dx.doi.org/10.1007/s10869-009-9140-2

Waldman, D. A., Balthazard, P. A., \& Peterson, S. J. (2011). Leadership and neuroscience: can we revolutionize the way that inspirational leaders are identified and developed? Academy of Management Perspectives, 25(1), 60-74. 
Watson, T. (2007). Organization theory and HRM. In P. Boxall, J. Purcell, \& P. Wright (Orgs.), The Oxford handbook of human resource management (pp. 108-127). New York: Oxford University Press.

Wei, L. (2006). Strategic human resource management: determinants of fit. Research and Practice in Human Resource Management, 14(2), 49-60.

Wright P. M., \& Boswell, W. R. (2002). Resource management research desegregating HRM: a review and synthesis of micro and macro human. Journal of Management, 28(3), 247-276. http://dx.doi.org/10.1177/014920630202800302

Wright, P. M., Gardner, T. M., Moynihan, L. M., \& Allen, M. R (2005). The relationship between HR practices and firm performance: examining causal order. Personnel Psychology, 58(2), 409-446. http://dx.doi.org/10.1111/j.1744-6570.2005.00487.x

Wright, P. M., \& McMahan, G. C. (1992). Theoretical perspectives for strategic human resource $\begin{array}{llll}\text { management. Journal of } & \text { 295-320. }\end{array}$ http://dx.doi.org/10.1177/014920639201800205

Wright, P. M., \& Snell, S. A. (1998). Toward a unifying framework for exploring fit and flexibility in strategic human resource management. Academy of Management Review, 23(4), 756-772. http://dx.doi.org/10.5465/AMR.1998.1255637

Yukl, G. (2012). Effective leadership behavior: what we know and what questions need more attention. Academy of Management Perspectives, 26(4), 66-85. http://dx.doi.org/10.5465/amp.2012.0088

Zhang, Y., Lepine, J. A., Buckman, B. R., \& Wei, F. (2014). It's not fair... or is it? The role of justice and leadership in explaining work stressor-job performance relationships. Academy of Management Journal, 57(3), 675-697. http://dx.doi.org/10.5465/amj.2011.1110

\section{Dados dos Autores}

Eliane Maria Pires Giavina Bianchi

Rua Guatemala, 167, Campo Limpo Paulista, 13231-230, São Paulo, SP, Brasil. E-mail: eliane.pires.bianchi@ terra.com.br

Alessandra Quishida

Rua Prof. Luciano Gualberto, 908, FEA-1 sala E-200, 05508-010, São Paulo, SP, Brasil. E-mail: quishida@gmail.com

Paula Gabriela Foroni

Rua Prof. Luciano Gualberto, 908, 05508-010, São Paulo, SP, Brasil. E-mail: pforoni@gmail.com 